\title{
Optimal strategies for tests of EPR-Steering with no detection loophole
}

Cite as: AIP Conference Proceedings 1633, 19 (2014); https://doi.org/10.1063/1.4903082

Published Online: 17 February 2015

David A. Evans, and Howard M. Wiseman
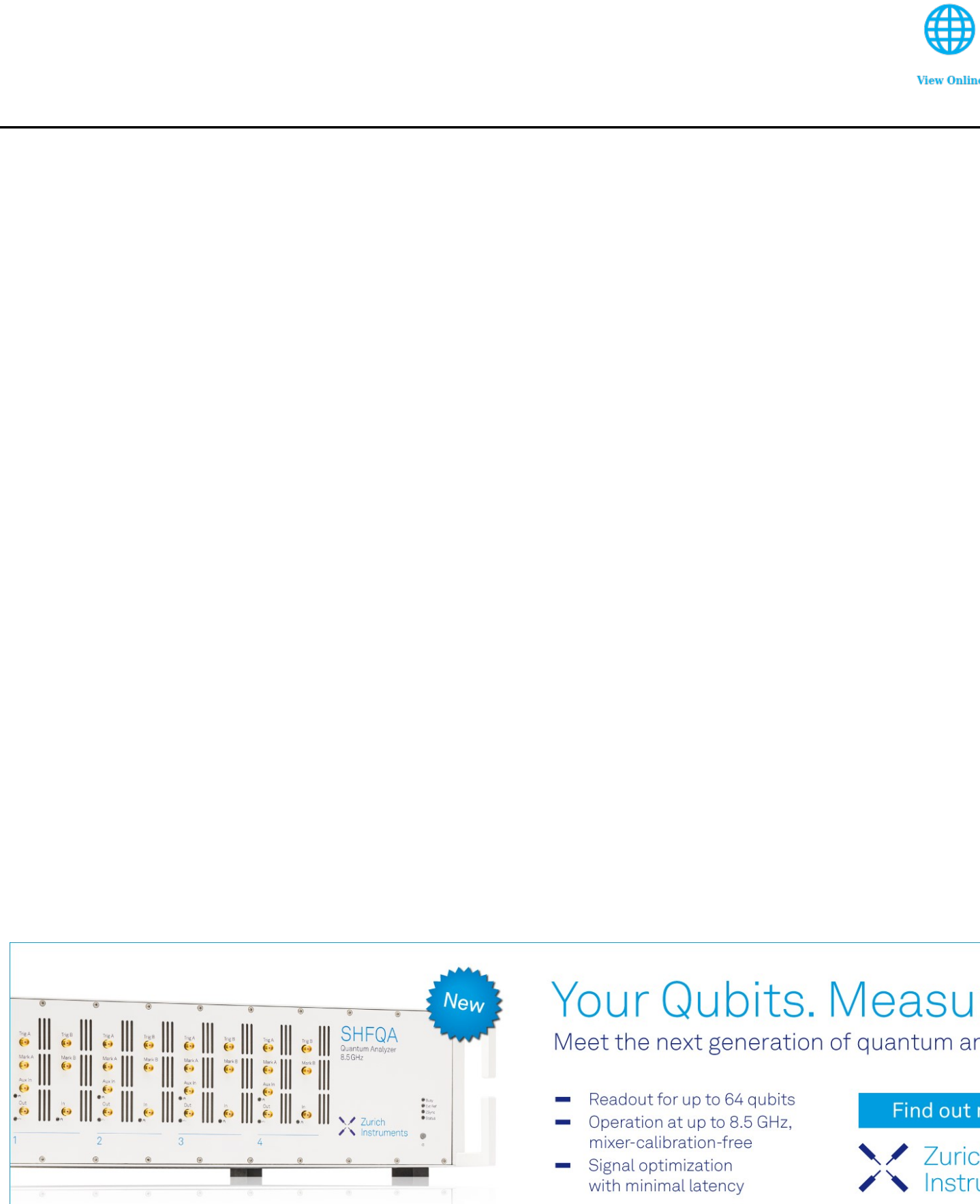

\section{Your Qubits. Measured.}

Meet the next generation of quantum analyzers

- Readout for up to 64 qubits

- Operation at up to $8.5 \mathrm{GHz}$.

mixer-calibration-free

- Signal optimization with minimal latency 


\title{
Optimal Strategies for Tests of EPR-Steering with No Detection Loophole
}

\author{
David A. Evans and Howard. M. Wiseman \\ Centre for Quantum Dynamics, Griffith University, Brisbane QLD 4111, Australia
}

\begin{abstract}
It has been shown in earlier works that the vertices of Platonic solids are good measurement choices for tests of EPR-Steering. Such measurements are regularly spaced, and measurement diversity is a good feature for making EPR-Steering inequalities easier to violate with entangled states. However, such measurements are provably suboptimal. By scrutinising not only the average results, but also the measurement statistics of cheating strategies in a Local Hidden State model, we outline a method for devising optimal strategies for EPR-Steering tests with no detection loophole.
\end{abstract}

Keywords: EPR, Steering, Detection Loophole

PACS: $03.65 \mathrm{Ud}, 42.50 \mathrm{Xa}$

\section{TESTS OF EPR-STEERING}

As demonstration of Bell nonlocality requires violation of a Bell inequality [1], so too does demonstration of EPR-Steering [2,3] require violation of EPR-Steering inequalities, which are less experimentally demanding than Bell inequalities. Tests of EPRSteering between two parties are such that one party's results are explicitly trusted, while the other is deemed untrustworthy $[4,5]$. Therefore, in such tests, we need only account for the inefficiency in one of the two detectors necessary for experimental execution of the test (in contrast to a loophole-free Bell inequality). When under a fair sampling assumption, a detection loophole is opened - the untrusted party (herein, Alice) can take advantage of a claimed detector inefficiency to falsely convince the other party (Bob) that she can steer his state. Closing such loopholes results in EPR-Steering inequalities becoming more difficult to violate experimentally. Thus, it is worthwhile to know which EPR-Steering bounds are the easiest to violate while still being free of loopholes.

\section{EPR-STEERING CRITERIA}

In our operational definition, Bob performs measurements on his qubit state and instructs Alice to make the same ones. Alice submits her results to Bob, who calculates an EPRSteering correlation function, and if it exceeds the maximum value obtainable using a Local Hidden State (LHS) model, then Bob is convinced that they share an entangled state, and that Alice has demonstrated EPR-Steering. The EPR-Steering correlations we use, $S_{n}$, are derived from the correlation function of Alice's reported results and Bob's measurement results:

$$
S_{n}=\frac{1}{n} \sum_{j}^{n} p_{j}\left\langle A_{j} \hat{\sigma}_{j}^{\beta}\right\rangle
$$

\footnotetext{
Eleventh International Conference on Quantum Communication, Measurement and Computation (QCMC) AIP Conf. Proc. 1633, 19-21 (2014); doi: 10.1063/1.4903082

(C) 2014 AIP Publishing LLC 978-0-7354-1272-9/\$30.00
} 
where $\hat{\sigma}_{j}^{\beta}$ are Bob's observables, $A_{j}$ are Alice's reported results on the same observables (unlike Bob's, we do not assume that they are actual measurement results), and $p_{j}$ are the weightings with which Bob chooses each measurement. The index $j$ is over the $n$ elements of Bob's measurement set. Bob considers that Alice may be obtaining her results through some LHS cheating strategy, rather than through spin measurements, like his. If Alice submits nulls for a portion $\varepsilon$ of her measurements, Bob also considers that this apparent efficiency may be part of a cheating strategy. Bob calculates the strategies that Alice is able to use to this end and, from them, defines bounds $k_{n}$ on $S_{n}$.

$$
k_{n}(\varepsilon)=\max _{\left\{w_{m}\right\}}\left[\sum_{m=1}^{n} w_{m} d_{n}\left(\varepsilon_{m}\right)\right], d_{n}\left(\varepsilon_{m}\right)=\frac{1}{\varepsilon_{m} n} \max _{\left\{A_{j}\right\}}\left[\lambda_{\max }\left(\sum_{j=1}^{n} p_{j} A_{j} \hat{\sigma}_{j}^{\beta}\right)\right],
$$

where $d_{n}\left(\varepsilon_{m}\right)$ bounds $S_{n}$ for a cheating Alice if she always chooses the same $m$ out of Bob's $n$ measurements to be null (so the apparent efficiency of $d_{n}\left(\varepsilon_{m}\right)$ is $\varepsilon_{m}=\frac{m}{n}$ ). But if she does not, then Alice's strategy will necessarily be a mixture of $d_{n}\left(\varepsilon_{m}\right)$ strategies, weighted by the set of $w_{m}$ values (which has constraints $\sum_{m}^{n} w_{m}=1$ and $\sum_{m}^{n} w_{m} \varepsilon_{m}=\varepsilon$ ).

Thus, from Alice's optimal cheating strategies, Bob defines his EPR-Steering inequalities, and is only convinced of the presence of EPR-Steering when Alice's results violate $S_{n} \leq k_{n}$, demonstrating that she does not possess the limitations of a LHS model.

Bob can calculate that such an inequality has a lower $k_{n}$ bound (and thus is easier for an honest Alice to violate) if he chooses measurements that are all as different as possible. An obvious choice is to make Bob's measurement orientations correspond to the vertices of Platonic solids, and to weight them equally. Based on this idea, Platonic solid measurements have previously been used to calculate EPR-Steering bounds in this format [6]. The 3D Platonic solids can be used to define measurement sets of size $n=2,3,4,6$, and 10. EPR-Steering bounds calculated using these measurements reveal that using more measurements generally gives more loss-tolerant bounds. Thus, one limitation when using Platonic solids is that $n \leq 10$. A way to get around this could be to use geodesic solids to define measurements which would not be equally spaced, but would be close to it, and could be constructed to employ arbitrarily many measurements [6]. EPR-Steering bounds calculated from the Platonic solids, and from the $n=16$ geodesic solid, are plotted in Figure 1a.

\section{OPTIMAL EPR-STEERING MEASUREMENTS}

The Platonic (and geodesic) measurements lead to rather loss-tolerant bounds, but one should notice in Figure 1a that near $\varepsilon=0.5$, the $n=3$ bound is lower than the $n=4$ bound, which should not occur if the $n=4$ bound was truly optimal. Therefore, the Platonic $n=4$ bound, at least, is definitely not an optimal EPR-Steering bound for four measurements. If it were, it would be at least as effective as the $n=3$ bound at all points.

To find measurements that are optimal for $n=4$, we must optimise Bob's measurement set (and measurement weightings, $p_{j}$ ) to attain the lowest value possible for Eq. 2. Performing this optimisation, we find that the optimal $n=4$ measurements are not of the $n=4$ platonic solid (the cube), but a shape with three vertex pairs almost equally spaced in one plane, and the fourth vertex perpendicular to them (with $p_{j}$ values that 

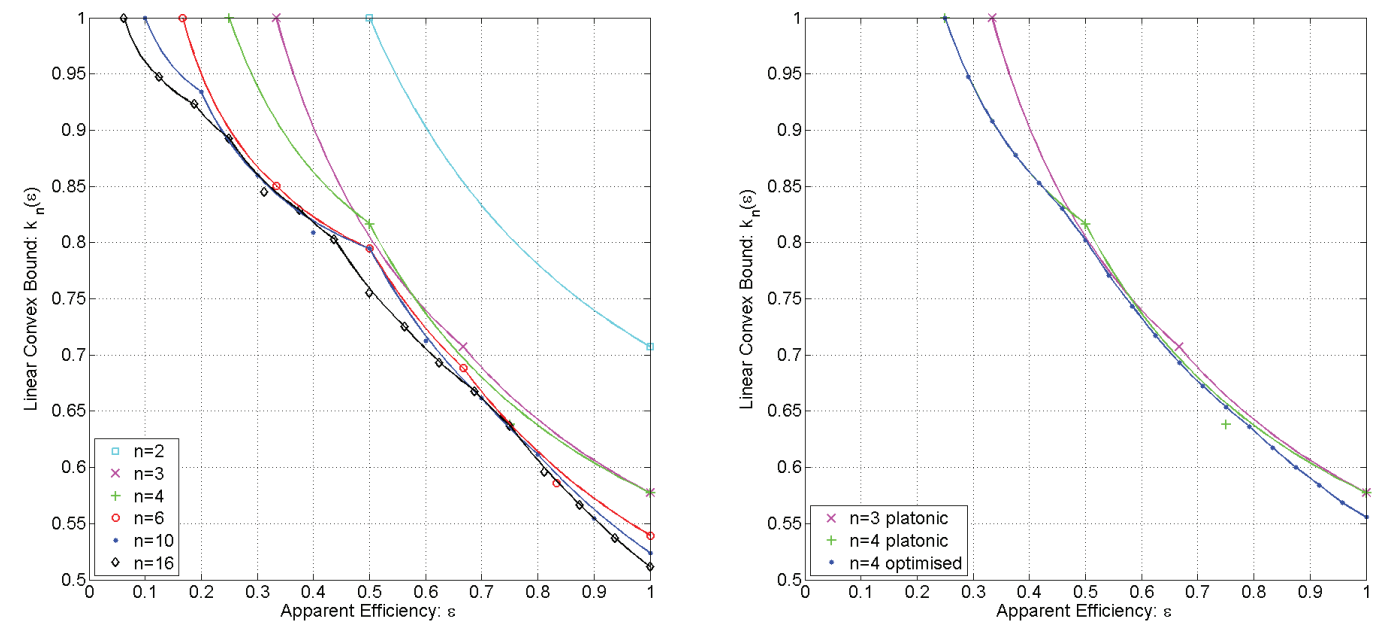

FIGURE 1. a) The Platonic and $n=16$ geodesic EPR-Steering bounds (left), and b) EPR-Steering bounds for optimised $n=4$ measurements, compared with Platonic $n=3$ and $n=4$ bounds (right).

are no longer all equal). The EPR-Steering bound attained with these measurements is shown in Figure $1 \mathrm{~b}$, and is equal to or less than the Platonic $n=4$ bound at all points. Optimising for $n=3$ reveals that the Platonic $n=3$ measurements actually are optimal.

\section{CONCLUSION}

Platonic solid measurement strategies are quite loss-tolerant, but are limited in number and are, in general, not optimal. We have calculated optimal strategies using three and four measurements, with optimisations that are not limited to any measurement number. Therefore, with these methods it is possible to derive measurement strategies for EPRSteering tests using any number of measurements. This is highly beneficial (especially for low detection efficiencies) since this means that more measurements can always yield more rigorous and more loss-tolerant bounds in tests of EPR-Steering.

\section{REFERENCES}

1. J. S. Bell, Physics 1, No. 3, 195 (1964).

2. A. Einstein, B. Podolsky, and N. Rosen, Physical Review 47, 777 (1935).

3. E. Schrödinger, Proc. Cambridge Philos. Soc. 31, 555 (1935).

4. H. M. Wiseman, S. J. Jones, and A. C. Doherty, Phys. Rev. Lett. 98, 140402 (2007).

5. E. G. Cavalcanti, S. J. Jones, H. M. Wiseman, and M. D. Reid, Phys. Rev. A 80, 032112 (2009).

6. A. J. Bennet, D. A. Evans, D. J. Saunders, C. Branciard, E. G. Cavalcanti, H. M. Wiseman, and G. J. Pryde, Phys. Rev. X 2, 031003 (2012).

7. B. Wittmann, S. Ramelow, F. Steinlechner, N. Langford, N. Brunner, H. Wiseman, R. Ursin, and A. Zeilinger, New J. Phys. 14, 053030 (2012).

8. D. H. Smith, G. Gillett, M. P. de Almeida, C. Branciard, A. Fedrizzi, T. J. Weinhold, A. Lita, B. Calkins, T. Gerrits, H. M. Wiseman, S. W. Nam, and A. G. White, Nat. Comm. 3, 625 (2012). 